\title{
Revisional Surgery after Failed Gastric Banding
}

\author{
Zullino Antonio*, Ravasio Giuditta, Califano Andrea, Asteria Carmela, Russo Emanuela and Giovanelli \\ Alessandro \\ Istituto Nazionale della Chirurgia dell'Obesità (INCO) \& Gruppo San Donato (GSD), Europe
}

Submission: August 10, 2017; Published: August 23, 2017

*Corresponding author: Antonio Zullino, Istituto Clinico Sant'Ambrogio, Via Faravelli 16-20149 Milan, Italy Tel: +39329062964;

Fax:+390233127076; Email: antonio.zullino@grupposandonato.it

\section{Abstract}

Background: Laparoscopic Adjustable Gastric Banding (LAGB) represents the first surgical approach used to treat morbid obesity. Due to both mechanical complications and/or inadequate weight loss, reoperation is often mandatory, in order to treat the band-related complications and to offer a better weight control. Objectives: We report our experience with revisional surgery after failed LAGB, comparing conversion to sleeve gastrectomy (SG), to Roux-en-Y gastric bypass (RYGB) and re-banding.

Setting: GSD (Gruppo San Donato) Hospitals, in Milan, Italy.

Methods: Between January 2000 and December 2014 all patients who underwent LAGB at our institution and who required surgical revision were retrospectively selected using a prospectively maintained database. A minimum post-revision follow-up of 24 months was observed.

Results: 1078 patients received LAGB and $99(9.1 \%), 85$ females and 14 males required revisional surgery after a mean period of 6 years: $71(72 \%)$ were reoperated for inadequate weight loss and 28 (28\%) due to a mechanical complication. After revisional surgery, mean BMI was reduced from $41.2 \pm 7.7$ to $31.4 \pm 5.5 \mathrm{Kg} / \mathrm{m}^{2}$ at 36 months and mean $\% E W L$ was increased up to $65.0 \pm 35.2 \%$ at 12 months, remaining > $60 \%$ until 36 months. Early complications occurred in 3 patients (3\%), late complications in 18 patients (18\%) with need of reoperation in 7 cases.

Conclusion: In our series LAGB was burdened by $9.1 \%$ of failure. Conversions to RYGB and SG produced similar improvement in weight control, with acceptable safety. Re-banding showed less weight control compared to first LAGB.

Keywords: HAdjustable gastric banding; Revisional bariatric surgery; Roux-en-Y gastric bypass; Sleeve gastrectomy; Band complications

\section{Introduction}

Laparoscopic Adjustable Gastric Banding (LAGB) was the first minimally invasive procedure for morbid obesity and has gained popularity for several decades due to its technical simplicity, reversibility; adjustability and relatively low surgical morbidity profile [1-3]. However, some studies have reported high rates of weight loss failure and band-related complications in the mid- and long term [3-5]. Band removal is mandatory in most cases, with conversion to different bariatric procedures necessary to face the recurrence of morbid obesity. Revisional surgery after failed LAGB is a debated problem, being the optimal choice and strategy of conversion unknown and often depending on the single case analysis, on the surgeon's skills and experience and on patient's will.

LAGB replacement or re-banding has been proposed by some authors but with limited success [6-8]. More extensive literature has been produced about LAGB conversion to laparoscopic sleeve gastrectomy (SG), to Roux-en-Y gastric bypass (RYGB) and to duodenal switch procedures [9-13]. In this study we aim to retrospectively evaluate the effectiveness of our revisional bariatric surgery after failed LAGB, in terms of Excess Weight Loss \% (EWL \%), Body Mass Index (BMI) course and complication rate.

\section{Methods}

We performed a review of our institutional database (prospectively maintained) between 2000 and 2014. We included all patients having a LAGB in our Centre, subsequently requiring revisional bariatric surgery with at least 2 years of follow-up.

\section{The indications for redo-surgery were}

Inadequate weight loss or weight regain, resulting in an EWL $\%<25 \%$, in absence of digestive symptoms or radiologic 
band displacement mechanical complications (slippage / pouch dilatation, trans-gastric migration, band leak, severe reflux): The following parameters were registered for each patient: demographics, indication to redo-surgery, time to revision and surgical strategy adopted, complications, BMI and EWL\% trend during follow-up visits. Each patient underwent endoscopy and contrast X-ray before surgery, and was routinely evaluated by dietist and psychologist. Revisional bariatric surgery was proposed after collegial analysis of the case and the indication was always advanced in observance of the current Italian Society for Bariatric and Metabolic Surgery (SICOB) guidelines [14].

\section{Operative technique}

All revisional bariatric surgery was performed by one bariatric surgeon who had performed over 1500 redo-bariatric procedures. The revisional procedure of choice was always discussed with our multidisciplinary team and after agreed with patient, depending on several factors, such as patient history, eating habits and preference, reason to redo-surgery, presence of metabolic comorbidities and intra-operative findings. All cases were operated by laparoscopy using four-five trocars: the band was completely freed by the hepato-gastric adhesions and the gastro-gastric stitches (if present) and then removed. The remaining scar ring below the band was interrupted with a vertical escharotomy to expose the gastric serosa. If the restoration of the normal gastric surgical anatomy was obtained and the gastric wall appeared suitable to be cut, onestep revisional surgery was performed. Our options were LAGB replacing / re-banding, conversion to SG and RYGB.

\section{Re-banding}

This option was considered when slippage with pouch dilatation or band leak occurred in good-LAGB compliant patients who experienced satisfying weight loss and were willing to re-banding. This operation was usually performed through four trocars: after band removal and complete gastric freeing from the adhesions with the left hepatic lobe, the new band was placed according to the pars flaccida approach trying to obtain a new retro-gastric route. In absence of surgical doubts, no pressure test was routinely executed during the intervention.

\section{Conversion to SG}

After removal of the band and dissection of the Angle of His with exposition of the left crus, the greater gastric curve was completely mobilized until $3-5 \mathrm{~cm}$ proximal to the pylorus and any posterior attachment was taken down. Then, a 38-French bougie was pulled down under direct visualization along the lesser curve to the level of the pylorus. SG was created by the Echelon Flex 60 (Ethicon Endosurgery, Cincinnati, OH, USA) using two initial gold or green cartridges and continuing with blue or gold cartridges respectively. No running suture was routinely performed along the gastric staple line. Methylene blue test was always executed and a tubular drain placed along the gastric section.

\section{Conversion to RYGB}

After band removal, the gastric horizontal transection was performed above or below the scar tissue of the band depending on the pouch volume above the band. Gold or green cartridges were used for gastric pouch creation, depending on the stomach wall thickness. Until 2015, a $70 \mathrm{~cm}$ bilio-pancreatic limb from the duodeno-jejunal flexure and a $100 \mathrm{~cm}$ alimentary limb were our standardized lengths as in primitive RYGB; since then, a $100 \mathrm{~cm}$ digestive and $150 \mathrm{~cm}$ alimentary limbs became our new standardized model of redo-RYGB. Stapled side-toside entero-enteral and antecolic gastro-jejunal anastomosis were performed with a single layer hand-sewn suture to close the common openings. Methylene blue was always executed to test the gastro-jejunal anastomosis and a laminar drain placed along the gastric pouch and anastomosis. Petersen's and Brolin's spaces were not routinely closed until 2014.

\section{Statistical analysis}

One-way ANOVA was computed in order to evaluate differences between three performed interventions. Furthermore, Cohen's d and its 95\% confidence interval (CI) were evaluated in order to explore the extent of differences among the surgical procedures in BMI and \%EWL.

\section{Results}

From January 2000 to December 2014, 1078 LAGB were performed as first bariatric procedure at our Bariatric Surgery Unit of INCO (Istituto Nazionale per la Chirurgia dell'Obesità) of GSD (Gruppo San Donato) Hospitals, in Milan, Italy. Among them, 99 patients (9.1\%), 85 females and 14 males with a mean age of 39 ( \pm 9 ) years, underwent revisional surgery after a mean period of $6.1( \pm 2.9$ ) years. Patients with LAGB placed in outside centers, referring to us for revisional surgery, were not included in this study. The indication was weight failure (inadequate weight loss or weight regain) in 71 cases and mechanical complications in 28 cases. Table 1 specifies the indications to redo-surgery. Seventysix patients underwent RYGB conversion (76.8\%), fifteen SG conversion (15.1\%) and 8 re-banding (8.1\%).

Table 1: Indication for revisional surgery after LAGB ( $n=99$ patients).

\begin{tabular}{|c|c|}
\hline & n (\%) \\
\hline Inadequate weight loss / regain & $71(72 \%)$ \\
\hline Mechanical complication & $28(28 \%)$ \\
\hline a) Slippage / pouch dilatation & $10(10 \%)$ \\
\hline b) Food intolerance (dysphagia, vomiting) & $10(10 \%)$ \\
\hline c) Trans-parietal band migration / erosion & $4(4 \%)$ \\
\hline d) Others (band leak, tube leak, shoulder \\
pain)
\end{tabular}

All interventions were laparoscopically performed with onestage conversion successfully obtained in 88 patients (88.8\%) and two-stages approach in the remaining 11 cases (10 for RYGB and 1 for SG conversion): 4 cases due to trans-parietal band migration (in which band removal was performed with 
combined endoscopic and laparoscopic approach), 4 cases due to caudal slippage with dilated and thinned pouch and 3 cases whose band removal was hard and long lasting. We experienced only 3 surgical complications (3\%) in the early postoperative period (within 30 days of surgery): 2 cases of abdominal bleeding conservatively treated and 1 one case of gastric fistula manifesting with septic manifestations on the 4 th postoperative day after SG, treated with laparoscopic conversion to RYGB. Late complications included: 7 cases of marginal ulcer, 6 Petersen's hernias, 1 occlusion by intestinal volvulus and 1 deep venous thrombosis following RYGB conversion (19.7\% of the cases), 2 cases of gastro-oesophageal reflux disease (with endoscopic signs of esophagitis) following SG conversion $(13.3 \%$ of the cases) and 1 incisional hernia after re-banding $(12.5 \%$ of the cases).

Table 2: Demographics and operative data.

\begin{tabular}{|c|c|c|c|}
\hline \multicolumn{4}{|c|}{ Demographics and operative data } \\
\hline Variable & All & RYGB & SG \\
\hline Total (female/male) & $99(85 / 14)$ & $76(68 / 8)$ & $15(11 / 4)$ \\
\hline Age (yr) & $39.3 \pm 8.8$ & $39.2 \pm 9.3$ & $39.4 \pm 7.2$ \\
\hline \multicolumn{3}{|c|}{ Indication to revision } \\
\hline i.Weight loss failure & 71 & 61 & 10 \\
\hline ii.Complication & 28 & 15 & 5 \\
\hline $\begin{array}{c}\text { Time to revision } \\
\text { (from AGB } \\
\text { placement) (yr) }\end{array}$ & $6.1 \pm 2.9$ & $6.5 \pm 2.9$ & $5.0 \pm 3.1$ \\
\hline $\begin{array}{c}\text { Mean follow-up after } \\
\text { revision (mo) }\end{array}$ & $41.2 \pm 7.7$ & $41.8 \pm 8.1$ & $37.9 \pm 5.6$ \\
\hline $\begin{array}{c}\text { Mean BMI at } \\
\text { revision (kg/m }{ }^{2} \text { ) }\end{array}$ & $34.7 \pm 6.9$ & $35.3 \pm 5.6$ & $32.9 \pm 6.2$ \\
\hline $\begin{array}{c}\text { Nadir BMI before } \\
\text { revision (kg/m }{ }^{2} \text { ) }\end{array}$ & $31.4 \pm 5.5$ & $31.3 \pm 5.3$ & $27.7 \pm 5.9$ \\
\hline $\begin{array}{c}\text { Nadir BMI after } \\
\text { revision (kg/m²) }\end{array}$ & (at $36 \mathrm{mo})$ & (at $24 \mathrm{mo})$ & (at 36 mo) \\
\hline $\begin{array}{c}\text { Maximum EWL } \\
\text { before revision (\%) }\end{array}$ & $50.6 \pm 30.5$ & $48.9 \pm 29.5$ & $52.5 \pm 35.9$ \\
\hline $\begin{array}{c}\text { Maximum EWL after } \\
\text { revision (\%) }\end{array}$ & $\begin{array}{l}65.0 \pm 35.2 \\
\text { (at } 12 \mathrm{mo})\end{array}$ & $\begin{array}{c}68.0 \pm 33.6 \\
\text { (at } 12 \mathrm{mo})\end{array}$ & $\begin{array}{l}95.0 \pm 48.7 \\
\text { (at } 36 \mathrm{mo})\end{array}$ \\
\hline
\end{tabular}

Table 2 shows the distribution of our study population according to the type of revisional surgery, demographic and anthropometric parameters before and after surgical revision. We found an overall mean BMI at revision of $41.2 \pm 7.7 \mathrm{Kg} /$ $\mathrm{m}^{2}$, significantly increased from the nadir mean BMI of $34.7 \pm$ $6.9 \mathrm{Kg} / \mathrm{m}^{2}$ registered at about 36 months from the first LAGB placement. After revisional surgery, mean BMI was reduced to $37.5 \pm 7.6,34.8 \pm 7.3,32.8 \pm 6.4,31.6 \pm 6.0,31.5 \pm 5.5$ and 31.4 $\pm 5.5 \mathrm{Kg} / \mathrm{m}^{2}$ after $1,3,6,12,24$ and 36 months, respectively. Subsequently, we observed a slowly progressive BMI increase (Figure 1). Mean EWL\% reached the highest value at 12 months after redo-surgery $(65.0 \pm 35.2 \%)$ and remained over $50 \%$ in all patients until 60 months of follow-up (Figure 2).

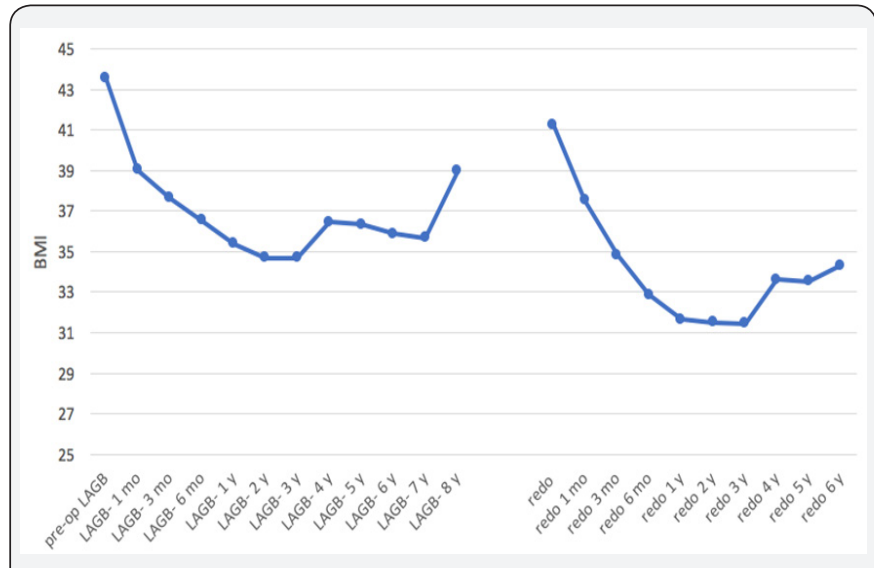

Figure 1: BMI curve before and after revisional surgery (preop: preoperative, mo: month, y: year).

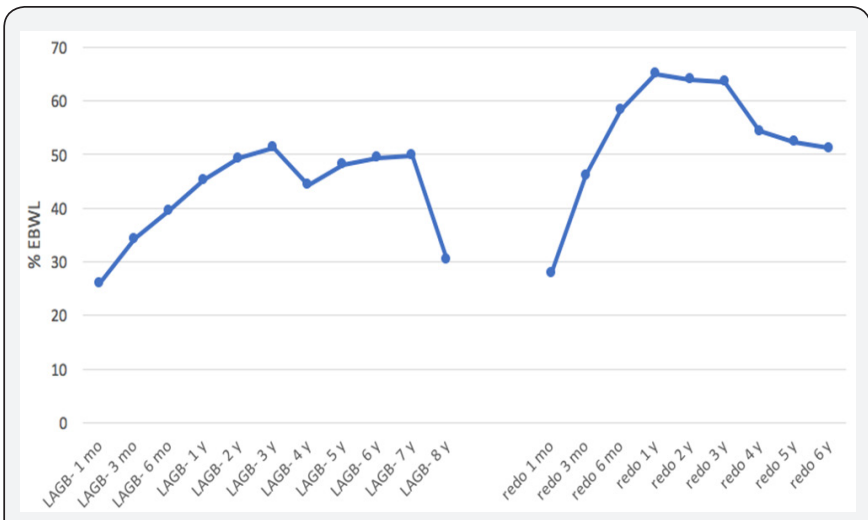

Figure 2: \%EWL curve before and after revisional surgery (mo: month, y: year).

All patients were evaluated at 1, 3, 6, 12, 24 months after revisional surgery, then the percentage of patients accessing the follow-up decreased to $81 \%$ (80 patients), 54\% (53 patients) and $30 \%$ (29 patients) at 36, 48 and 60 months, respectively. Mean BMI at the time of revisional surgery was not statistically different among the patients assigned to the three types of redosurgery $(F(2.95)=2.79 ; p=0.07)$. Figures $3-4$ respectively illustrate post-revisional BMI and EWL\% course for each type of redo-surgery. Relevant advantages in EWL\% were demonstrated in patients who underwent to revisional SG and RYGB, with large $(d \geq 0.80)$ statistical significance at 12 and 24 months for SG compared to re-banding $(\mathrm{d}=-1.07[-1.98--0.16]$ and $\mathrm{d}=-0.95$ [-1.95 - -0.05], respectively) and at 6 and 12 months for RYGB versus re-banding $(d=-0.90[-1.64--0.16]$ and $d=-0.80[-1.58$ - -0.06], respectively). Simultaneously, reductions in BMI were greater in patients who underwent revisional SG and RYGB rather than re-banding, but no statistical significance was found. Revisional SG and RYGB showed stronger weight loss in the same patients initially treated with LAGB, as demonstrated by lower mean BMI (SG: $27.7 \pm 5.8$ vs $32.9 \pm 6.2 \mathrm{Kg} / \mathrm{m}^{2} \mathrm{~d}=-0.86[-1.70$ $--0.02]$, RYGB: $31.3 \pm 5.3$ vs $35.3 \pm 7.2 \mathrm{Kg} / \mathrm{m}^{2}, \mathrm{~d}=-0.62[-0.96-$ -0.28]) and maximum mean EWL\% (SG: 94.548 .1 vs $52.5 \pm 35.8$ 
$\%, d=1.02$ [0.17 - 1.87], RYGB: $68.0 \pm 33.6$ vs $48.9 \pm 29.6 \% d=$ $0.60[0.26-0.94]$ ) (Figures $3 \& 4$ ).

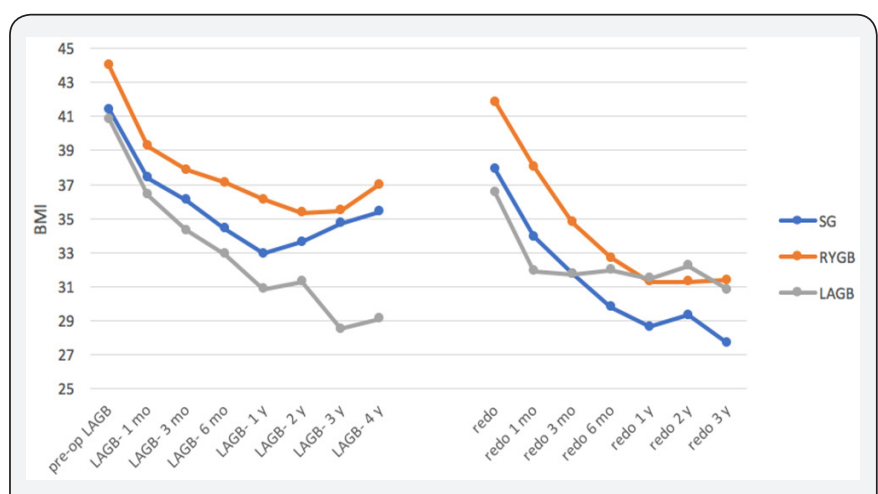

Figure 3: BMI curve before and after revisional surgery, stratified by type of redo-surgery (preop: preoperative, mo: month, y: year).

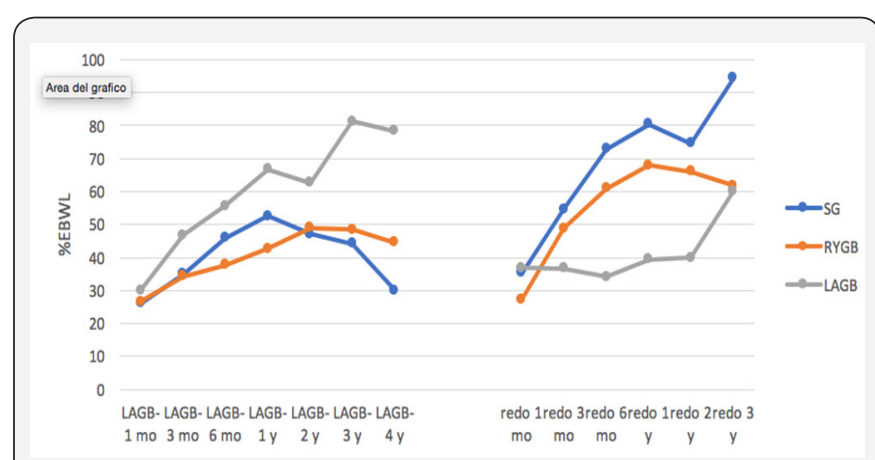

Figure 4: \%EWL curve before and after revisional surgery, stratified by type of redo-surgery (preop: preoperative, mo: month, y: year).

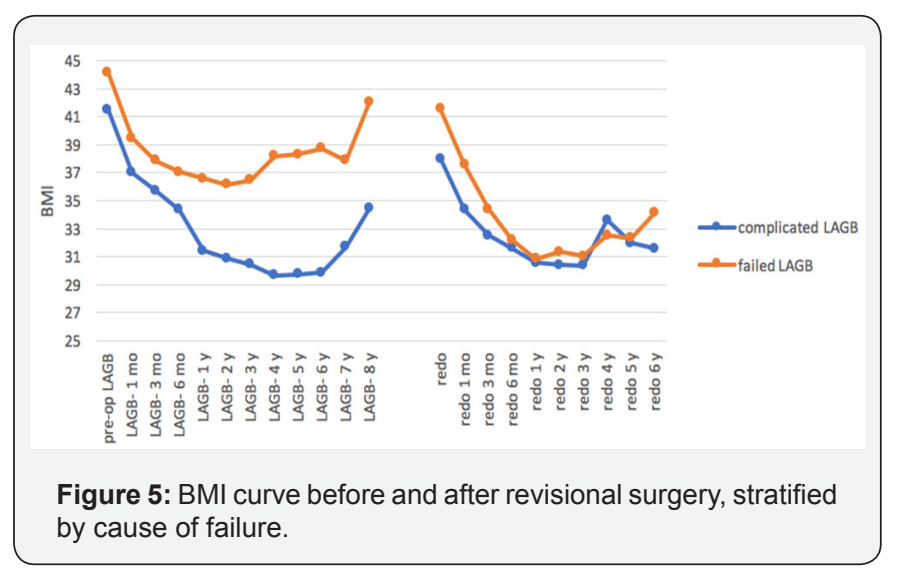

On the contrary, patients underwent re-banding did not experience the same effectiveness of the first LAGB (better BMI: $30.8 \pm 5.7$ vs $28.5 \pm 3.2 \mathrm{Kg} / \mathrm{m}^{2} \mathrm{~d}=0.50$ [-0.65 - 1.65]), maximum EWL $\% 60.1 \pm 20.7$ vs $81.2 \pm 17.2 \% \mathrm{~d}=-1.10$ [-2.32 - -0.11$]$ ). As expectable, a statistically different weight course was observed between poor-responder and complicated patients. The first never reached a mean \%EWL of $50 \%$. Moreover, a significant difference was found also in terms of nadir mean BMI and BMI at revision (nadir BMI: $29.6 \pm 3.6(n=14)$ at 48 months vs $36.1 \pm 6.7$
( $\mathrm{n}=64) \mathrm{Kg} / \mathrm{m}^{2}$ at 24 months, $\mathrm{d}=-1.03[-1.63--0.43]$ and BMI at revision: $37.9 \pm 6.5(\mathrm{n}=28)$ vs $41.5 \pm 7.8 \mathrm{Kg} / \mathrm{m}^{2}(\mathrm{n}=71), \mathrm{d}=-0.48$ [-0.92 - -0.03]), but these differences disappeared after only 3 months from revisional surgery (Figure 5).

\section{Discussion}

LAGB was the first minimally invasive surgical approach to morbid obesity, with satisfying results in terms of early weight loss and morbidity, and with almost no mortality. The initial enthusiasm for LAGB went progressively lessening due to the increasing number of studies focused on its long-term followup. Weight loss failure or regain and high rates of band-related complications were reported by several authors [5,15-18] and may be considered the most important drawback of this procedure. Our data are in agreement with others reported in literature. In our series, the most common indication to reoperation was weight loss failure and regain, registered in 71 (72\%) out of 99 patients.

The remaining 28 patients (28\%) required operative reintervention due to band complications, from 6 to 72 months after the first LAGB placement. It is important to underline that different surgical scenarios are possible under the item "bandrelated complications". While most of them can be easily solved and directly converted to another bariatric intervention, in some cases the anatomic exposure following the band retrieval may not be satisfactory and it may suggest postponing the revisional surgery. Nevertheless, it should be remembered that some patients report a good experience with LAGB, with long-lasting weight control and without any long-term complications. We calculated that $9.1 \%$ of all patients firstly receiving LAGB at our Centre have subsequently referred to us for revisional surgery, although this percentage underestimates the real amount of our failed LAGB because of a high number of patients lost during the follow-up and many others with inadequate weight response not asking for redo-surgery.

The aim of revisional bariatric surgery after LAGB is to treat the band-related complications and to ensure a better control on weight and the eventually concomitant obesity comorbidities. There is no universally accepted algorithm regarding the reoperative treatment after failed LAGB. RYGB conversion is supported by the most part of the authors, but also other options are contemplated, such as conversion to SG or to malabsorptive procedures. We agree with the theory that a failed response to a restrictive procedure should be faced with a not purely restrictive one [6], unless those few cases where a mild band complication (e.g., slippage with no huge gastric pouch, band leak, tube leak) could be considered the only cause for LAGB failure. Whenever possible, we opted for RYGB conversion (about $77 \%$ of patients in the present series). When it did not meet the patient's preference or some contraindications to RYGB coexisted (finding of intestinal metaplasia or dysplasia in endoscopic biopsy of gastric mucosa, antibiotic-resistance to 
Helicobacter pylori infection, and strong familiarity for gastric carcinoma), SG was taken into account as possible alternative.

However, it is important to keep in mind that the risk of gastric leak is reported to be higher after conversion from LAGB to SG [19-21]. In our series, we had only one case of leak developed from the staple line at the level of the scar ring after LAGB removal and sleeve resection at the same time. Its early presentation and timely diagnosis allowed us to perform a successful conversion to RYGB. Finally, re-banding was performed in eight patients, with previous good comfort with the device, asking for a second LAGB after a mild band complication that interrupted a satisfactory weight loss. Our data seem to suggest that revisional bariatric surgery is a reasonable instrument in order to ensure good weight control after failed LAGB, with acceptable safety. We obtained a significant mean BMI reduction up to 10 points at 36 months from redo-surgery and an increasing of \%EWL up to the highest mean value of $65.0 \pm 35.2 \%$ at 12 months from revision, resulting $>60 \%$ until 36 months and $>50 \%$ during all subsequent follow-up (Figures 1 \& 2).

When the revisional interventions are separately analysed, both RYGB and SG resulted to be superior to re-banding in terms of weight loss outcomes. This is statistically evident when EWL\% curves are considered: RYGB and SG showed a mean EWL $\%>60 \%$ between 6 and 36 months after surgery. We did not find any statistically significant difference between RYGB and SG. Otherwise, re-banding did not carry any advantages in weight control compared to the first LAGB, but ensured weight stability between 31 and $33 \mathrm{Kg} / \mathrm{m} 2$ in all patients. Although revisional bariatric surgery is technically more challenging compared to primary bariatric surgery, in the present series we had a low complication rate in the early postoperative period (about $3 \%$ ), less than other reported in literature [22-25].

Moreover, we observed $18 \%$ of complications in the late postoperative period, the most part of them being marginal ulcers (often in heavy smokers and drinkers) and Petersen's hernias until the closure of mesenteric spaces after RYGB was not routinely performed. In conclusion, LAGB is demonstrated to be associated with a relevant incidence of weight loss failure and potential long-term complications, which together explain the high reoperation rates reported in literature [7,9-11]. LAGB, more than other interventions, requires a high degree of patient compliance, including major changes in life style and eating habits, so it should be performed only in carefully selected patients, who prefer or who are more suitable for a less invasive and reversible surgical procedure.

After failed LAGB, different surgical options are possible, among them conversion to RYGB and SG have been historically taken into account in our clinical practice, both for satisfactory weight control and low-morbidity profile. Also malabsorptive interventions (bilio-pancreatic diversion with or without duodenal switch) were offered following failure of LAGB by some authors [10,26-28], but up to now, these procedures have never been our choice, due to the relevant risks of malnutrition and vitamin/mineral deficiency. This article adds a contribution to the current literature about revisional bariatric surgery following LAGB failure, but larger series and longer followups are still needed to draw definitive conclusions. Our study has some limitations: firstly, it is a retrospective, mono-centric and not randomized study. Moreover, the study population is small and composed by unbalanced little subgroups of patients that can affect the statistical significance of the comparisons. Finally, we did not evaluate the impact of the different bariatric interventions on obesity-related morbidities, medication used and quality of life.

\section{Conflict of Interest Disclosure Statement}

The authors declare that they have no conflict of interest.

\section{Acknowledgement}

We would like to thank Dr M Cavicchioli for statistical analysis and Dr C Gallo Stampino for supporting the collection of our data. This was of great importance for developing this article.

\section{References}

1. Saber AA, Elgamal MH, McLeod MK (2008) Bariatric surgery: the past, present, and the future. Obes Surg 18(1): 121-128.

2. Khoursheed MA, Al-Bader IA, Al-Safar FS, Mohammad AL, Shukkur M, et al. (2011) Revision of failed bariatric procedure to Roux-en-Y gastric bypass (RYGB). Obes Surg 21(8): 1157-1160.

3. van Wageningen B, Berends FJ, van Ramshorst B, Janssen IMC (2006) Revision of failed laparoscopic adjustable gastric banding to Roux-en-Y gastric bypass. Obes Surg 16(2): 137-141.

4. Suter M, Calmes JM, Paroz A, Giusti V(2006) A 10-year experience with laparoscopic gastric banding for morbid obesity: high long-term complication and failure rates. Obes Surg 16(7): 829-835.

5. Allen JW (2007) Laparoscopic gastric band complications. Med Clin N Am 91(3): 485-497.

6. Weber M, Muller MK, Michel JM, Belal R, Horber F et al. (2003) Laparoscopic Roux-en-Y gastric bypass, but not rebanding, should be proposed as rescue procedure for patients with failed laparoscopic gastric banding. Ann Surg 238(6): 827-833

7. Muller MK, Attigah N, Wildi S, Hahnloser D, Hauser R, et al. (2008) High secondary failure rate of rebanding after failed gastric banding. Surg Endosc 22(2): 448-453.

8. te Riele WW, van Santvoort HC, Boerma D, van Westreenen HL, Wiezer MJ, et al. (2014) Rebanding for slippage after gastric banding: should we do it? Obes Surg 24(4): 588-593.

9. Elnahas A, Graybiel K, Farrokhyar F, Gmora S, Anvari M, et al. (2013) Revisional surgery after failed laparoscopic adjustable gastric banding: a systematic review. Surg Endosc 27(3): 740-745

10. Gagner M, Gumbs AA (2007) Gastric banding: conversion to sleeve, bypass, or DS. Surg Endosc. 21(11): 1931-1935.

11. Suter M, Giusti V, Heraief E, Calmes JM (2004) Band erosion after laparoscopic gastric banding: occurrence and results after conversion to Roux-en-Y gastric bypass. Obes Surg 14(3): 381-386. 
12. Mognol P, Chosidow D, Marmuse JP (2004) Laparoscopic conversion of laparoscopic gastric banding to Roux-en-Y gastric bypass: a review of 70 patients. Obes Surg 14(10): 1349-1353.

13. Abu-Gazala S, Keidar A (2012) Conversion of failed gastric banding into four different bariatric procedures. Surg Obes Relat Dis 8(4): 400-407.

\section{4. www.sicob.org}

15. Chevallier JM, Zinzindohoué F, Douard R (2004) Complications after laparoscopic adjustable gastric banding for morbid obesity: experience with 1000 patients over 7 years. Obes Surg 14(3): 407-414.

16. Holéczy P, Novak P, Kralova A (2001) 30\% complications with adjustable gastric banding: what did we do wrong? Obes Surg 11(6): 748-751.

17. Peterli R, Donadini A, Peters T, Ackermann C, Tondelli P (2002) Reoperation following laparoscopic adjustable gastric banding. Obes Surg 12(6): 851-856.

18. Favretti F, Cadiere GB, Segato G (1997) Laparoscopic adjustable silicone gastric banding (Lap-Band): how to avoid complications. Obes Surg 7(4): 352-358

19. Park YH, Kim SM (2014) Laparoscopic sleeve gastrectomy as revisional surgery for adjustable gastric band erosion. J Laparoendosc Adv Surg Tech A 24(9): 593-600.

20. Foletto M, Prevedello L, Bernante P et al. (2010) Sleeve gastrectomy as revisional procedure for failed gastric banding or gastroplasty. Surg Obes Relat Dis 6(2):146-151.
21. Iannelli A, Schneck AS, Ragot E (2009) Laparoscopic sleeve gastrectomy as revisional procedure for failed gastric banding and vertical banded gastroplasty. Obes Surg 19(9): 1216-1220.

22. Tran TT, Pauli E, Lyn-Sue JR, Haluck R, Rogers AM (2013) Revisional weight loss surgery after failed laparoscopic gastric banding: an institutional experience. Surg Endosc 27(11): 4087-4093.

23. Moore R, Perugini R, Czerniach D, Gallagher-Dorval K, et al. (2009) Early results of conversion of laparoscopic adjustable gastric band to Roux-en-Y gastric bypass. Surg Obes Relat Dis 5(4): 439-443.

24. Hii MV, Lake AC, Kenfield C, Hopkins GH (2012) Laparoscopic conversion of failed gastric banding to Roux-en-Y gastric bypass: shortterm follow-up and technical considerations. Obes Surg 22(7): 10221028.

25. Abdelgawad M, De Angelis F, Iossa A, Rizzello M, Cavallaro G, etal. (2016) Management of complications and outcomes after revisional bariatric surgery: 3-year experience at a bariatric center of excellence. Obes Surg 26(9): 2144-2149.

26. de Csepel J, Quinn T, Pomp A, Gagner M (2002) Conversion to a laparoscopic biliopancreatic diversion with a duodenal switch for failed laparoscopic adjustable silicone gastric banding. J Laparoendosc Adv Surg Tech A 12(4):237-240.

27. Dolan K, Fielding G (2004) Biliopancreatic diversion following failure of laparoscopic adjustable gastric banding. Surg Endosc 18(1): 60-63.

28. Daskalakis M, Scheffel O, Theodoridou S, Weiner RA (2009) Conversion of failed vertical banded gastroplasty to biliopancreatic diversion, a wise option. Obes Surg 19(12): 1617-1623.

\section{Your next submission with Juniper Publishers will reach you the below assets}

- Quality Editorial service

- Swift Peer Review

- Reprints availability

- E-prints Service

- Manuscript Podcast for convenient understanding

- Global attainment for your research

- Manuscript accessibility in different formats

( Pdf, E-pub, Full Text, Audio)

- Unceasing customer service

Track the below URL for one-step submission https://juniperpublishers.com/online-submission.php 